\title{
Determination of the of rate cross slip of screw dislocations
}

Vegge, Tejs; Rasmussen, Torben; Leffers, Torben; Pedersen, Ole Bøcker; Jacobsen, Karsten Wedel

Published in:

Physical Review Letters

Link to article, DOI:

10.1103/PhysRevLett.85.3866

Publication date:

2000

Document Version

Publisher's PDF, also known as Version of record

Link back to DTU Orbit

Citation (APA):

Vegge, T., Rasmussen, T., Leffers, T., Pedersen, O. B., \& Jacobsen, K. W. (2000). Determination of the of rate cross slip of screw dislocations. Physical Review Letters, 85(18), 3866-3869.

https://doi.org/10.1103/PhysRevLett.85.3866

\section{General rights}

Copyright and moral rights for the publications made accessible in the public portal are retained by the authors and/or other copyright owners and it is a condition of accessing publications that users recognise and abide by the legal requirements associated with these rights.

- Users may download and print one copy of any publication from the public portal for the purpose of private study or research.

- You may not further distribute the material or use it for any profit-making activity or commercial gain

- You may freely distribute the URL identifying the publication in the public portal 


\title{
Determination of the of Rate Cross Slip of Screw Dislocations
}

\author{
T. Vegge, ${ }^{1,2}$ T. Rasmussen, ${ }^{3}$ T. Leffers, ${ }^{2}$ O. B. Pedersen, ${ }^{2}$ and K. W. Jacobsen ${ }^{1}$ \\ ${ }^{1}$ Center for Atomic-scale Materials Physics (CAMP) and Department of Physics, Building 307, Technical University of Denmark, \\ DK-2800 Kgs. Lyngby, Denmark \\ ${ }^{2}$ Materials Research Department, Ris $\phi$ National Laboratory, DK-4000 Roskilde, Denmark \\ ${ }^{3}$ Theoretical Division, Los Alamos National Laboratory, Los Alamos, New Mexico 87545
}

(Received 16 June 2000)

\begin{abstract}
The rate for cross slip of screw dislocations during annihilation of screw dipoles in copper is determined by molecular dynamics simulations. The temperature dependence of the rate is seen to obey an Arrhenius behavior in the investigated temperature range: $225-375 \mathrm{~K}$. The activation energy and the effective attempt frequency can therefore be extracted from the simulations. The transition state energy for the annihilation process is calculated by identifying the transition state using the nudged elastic band path technique. The two activation energies agree very well, indicating that transition state theory is applicable for this type of process.
\end{abstract}

PACS numbers: 61.72.Lk, 07.05.Tp, 82.20.Db

To what extend can a simple Arrhenius rate law be used to describe elementary processes in materials? This question is of increasing relevance as modeling of material processes based on atomistic input is carried further. Direct molecular dynamics (MD) simulations can be used to address aspects of material behavior over short time scales as exemplified by studies of dislocation dynamics [1] or deformation of nanocrystalline metals [2]. However, modeling over longer times commonly resorts to an effective description of the atomistic dynamics in terms of a number of "events" with prescribed rates associated with them, where the rates are typically assumed to obey an Arrhenius temperature dependence. This approach has, for example, been used extensively in kinetic Monte Carlo simulations of diffusion and growth [3], and it is also fundamental in coarse-scale modeling of dislocation dynamics [4]. Rate theory is also an important ingredient in new accelerateddynamics techniques [5].

The applicability of the Arrhenius law - and the appropriate determination of activation energies and rate prefactors - has been dealt with in some detail in the case of simple atomic diffusion processes involving only a few atoms. Several studies have shown that harmonic transition state theory [6] is often applicable even at rather high temperatures $[3,7]$. Dislocation processes, on the other hand, are of a different nature. Dislocations are extended line defects and their motion involves the collective motion of many atoms. It is therefore from the outset unclear whether anharmonic entropic effects or dynamical recrossings may give rise to strongly temperature dependent prefactors. In the present paper we investigate the annihilation of a screw dislocation dipole by cross slip in copper, using both molecular dynamics simulations and rate theory, and show that the obtained activation energies agree completely. Also, the rate prefactor for cross slip determined from the MD simulations is found to have a typical atomistic value, in agreement with a theoretical estimate.
We focus on dislocation dipoles because they are considered to play an important role in the plastic deformation of fcc metals. The two determining factors in the dislocation density of deformed metals are the dislocation multiplication and annihilation rates. At ambient temperature, where climb processes are limited, dislocation annihilation occurs only via cross slip of screw dislocation [8]. In this Letter we investigate the activated annihilation of a dipole with height $h=1.3 \mathrm{~nm}$. The atomic interactions are calculated using an effective medium theory (EMT) many-body potential [9], which describes the elastic properties of copper quite well. The elastic constants are within $10 \%$ of experimental reference values, and the intrinsic stacking fault energy of the potential is $31 \mathrm{~mJ} / \mathrm{m}^{2}$, somewhat lower than the value $45 \mathrm{~mJ} / \mathrm{m}^{2}$ inferred from experiments [10] or $56 \mathrm{~mJ} / \mathrm{m}^{2}$ obtained with density functional theory [11].

The computational system used in the simulations contains 130680 atoms placed in a cell with $66\{111\}$ planes $(14.5 \mathrm{~nm})$ wide, nonorthogonal closed packed sides along the $(\overline{1} 11)$ and $(1 \overline{1} 1)$ planes. The cell extends $\ell=30 \mathbf{b}$ $(7.6 \mathrm{~nm})$ along the [110] direction - previously shown to be sufficient to describe the 3D nature of the annihilation process [12] — and we apply periodic boundary conditions in all directions. The two screw dislocations with opposite Burgers vectors, $\mathbf{b}= \pm \frac{a}{2}[110]$, are introduced into the cell as Shockley partial dislocations: $\alpha \mathrm{C}=\frac{a}{6}[21 \overline{1}]$ and $\mathrm{D} \alpha=\frac{a}{6}$ [121], and $\mathrm{C} \alpha$ and $\alpha \mathrm{D}$, respectively. The initial splitting of the partials is determined by linear, isotropic elasticity theory. The dipolar configuration, with the dislocations separated by six (111) planes $(1.3 \mathrm{~nm})$, is subsequently relaxed to a minimum energy configuration using the MDmin algorithm [13].

In the MD simulations the system is heated to a given temperature $T$ using Langevin dynamics [13] for a period of $t_{\text {heat }}=10 \mathrm{ps}$ [see frame (a) in Fig. 1]. Subsequently, the thermalized system is subjected to Verlet dynamics [13] until the dislocation annihilation has occurred. 


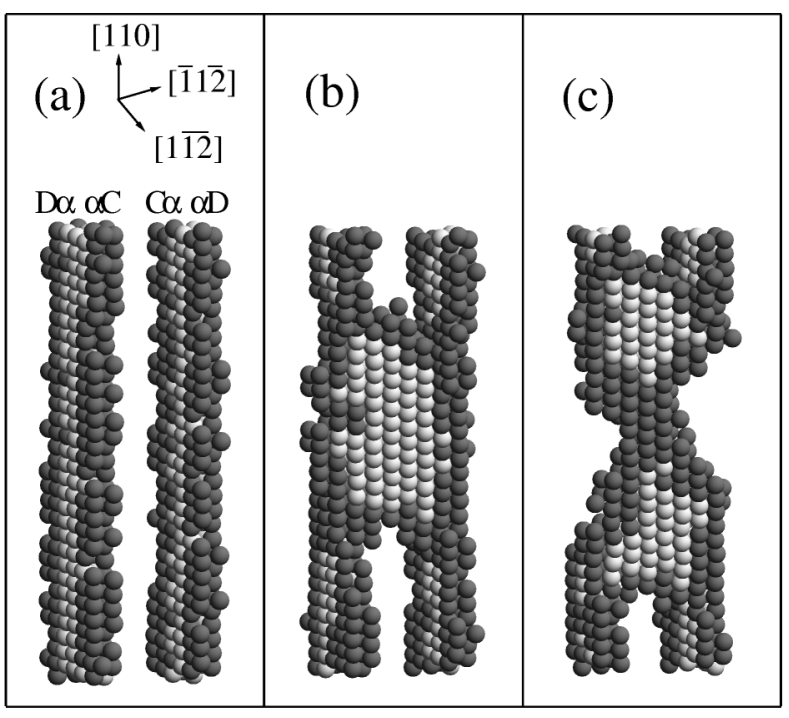

FIG. 1. Snapshots from the MD simulation of screw dipole annihilation at $350 \mathrm{~K}$. The atoms are colored in accordance with their neighbor relations: atoms within the Shockley partials are dark grey, atoms within stacking faults are light grey, and ordinary bulk atoms are invisible. Panel (a) shows the configuration after thermalization. In (b) cross slip has been initiated. In (c) two partials have annihilated along part of the (former) dipole, leaving a stacking fault between the remaining partials.

The point of annihilation can be clearly determined from the disappearance of the $\sigma_{[110][1 \overline{12}]}$ stress component in the simulation, as seen in the inset of Fig. 2 for $T=255 \mathrm{~K}$. The annihilation can be verified by performing a series of energy minimizations with initial conditions along the MD trajectory. At times earlier than $t=598.2 \mathrm{ps}$ the system falls into the initial state with the dislocation dipole, but at later times the system relaxes into the perfect crystal without any dislocations, i.e., no recrossings were observed.

We are therefore able to determine the time at which the annihilation event occurs (which we shall call the "lifetime" of the dipole in the following) as the time elapsed after the Langevin thermalization until the $\sigma_{[110][1 \overline{12}]}$ stress begins to disappear. The disappearance of the dislocation dipole can also be monitored by using the common neighbor analysis [14], as shown in Fig. 1.

The annihilation process could for this particular dipole height be studied with molecular dynamics in the temperature range from 225 to $375 \mathrm{~K}$. The lower temperature bound is determined by computational limitations, corresponding to more than $670000 \mathrm{MD}$ time steps ( $\sim 3 \mathrm{~ns})$. The upper bound corresponds to approximately 1700 time steps $(\sim 8 \mathrm{ps})$, since higher well-defined temperatures are inaccessible due to annihilation during the initial thermalization process.

We have performed a total of $19 \mathrm{MD}$ runs in the mentioned temperature range and for each run we have determined the lifetime of the dipole. The obtained lifetimes are shown on a logarithmic scale in Fig. 2 as a function of inverse temperature. Assuming that the lifetime $t$ is

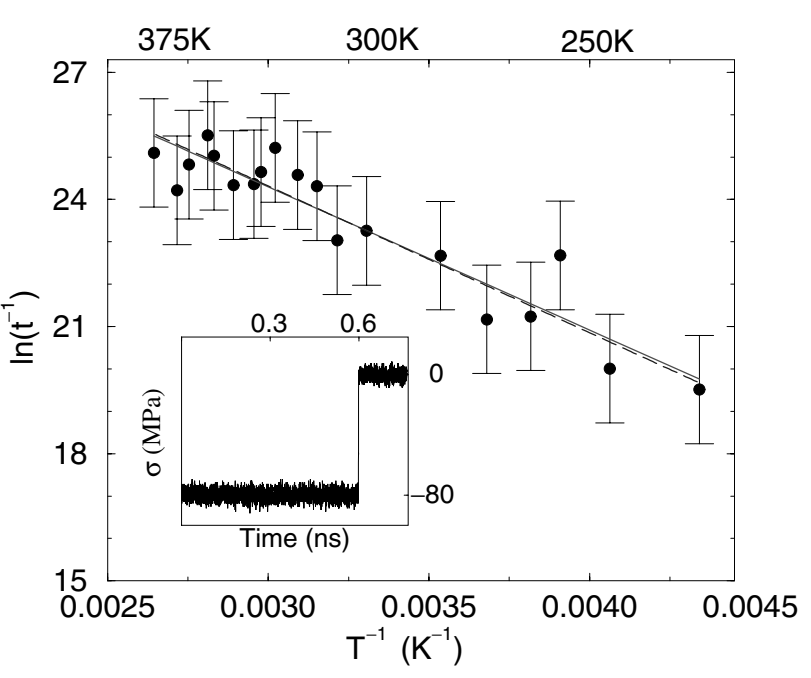

FIG. 2. Arrhenius plot of the annihilations times (lifetimes) for temperatures between 225 and $375 \mathrm{~K}$. The error bars represent the width of the thermal distribution. The solid line is a linear fit to the 19 data points, yielding an activation energy of $291 \pm$ $27 \mathrm{meV}$ and a $\nu$ value of $\left(4 \times 10^{13} \mathrm{sec}^{-1}\right) \mathbf{b}^{-1}$. The dashed line is the best fit to the data points for a line with slope corresponding to the zero Kelvin NEB activation energy of $315 \mathrm{meV}$. It yields a $\nu$ value of $\left(5 \times 10^{13} \mathrm{sec}^{-1}\right) \mathbf{b}^{-1}$. The inset shows the $\sigma_{[110][1 \overline{12}]}$ component as a function of time for the MD simulation at $255 \mathrm{~K}$

exponentially distributed at a given temperature, we have for the average of the logarithm of the lifetime $\langle\ln (t)\rangle=$ $\ln (\tau)-\gamma$, where $\tau$ denotes the average lifetime $\tau=\langle t\rangle$ and $\gamma$ is Euler's constant $(\gamma \approx 0.5772)$. The width $\sigma(\ln (t))$ of the distribution of $\ln (t)$ is given by $\sigma(\ln (t))=\pi / \sqrt{6} \approx$ 1.28 and is indicated in the figure as error bars.

It is seen that the data points in Fig. 2 can be fitted well with a straight line corresponding to an Arrhenius expression for the annihilation rate $r=1 / \tau$ :

$$
r=1 / \tau=\nu \exp \left(-E / k_{B} T\right),
$$

where $E$ denotes the activation energy. A least squares fit leads to the value $E=291 \pm 27 \mathrm{meV}$ of the activation energy and the prefactor can be estimated to be $\nu=2 \times$ $10^{15} \mathrm{sec}^{-1}$ for this dislocation length.

The MD results apparently follow the simple Arrhenius behavior in the investigated temperature range and we proceed to analyze the results using transition state rate theory (TST) [6]. In TST the rate can be written as

$$
r=\sqrt{\frac{T}{2 \pi m}} \exp \left(-\Delta F / k_{B} T\right),
$$

where $m$ is the atomic mass, and $\Delta F$ is the difference in free energy between the dividing surface (through the transition state) and the initial state. In the simplest harmonic approximation to TST [6], the Arrhenius form for the rate [Eq. (1)] is obtained, and the activation energy $E$ is identified as the difference between the energy in the transition state and the initial state. We therefore need to find the transition state (i.e., the saddle point on the potential energy surface) and we have done this by using the nudged 
elastic band (NEB) method [15]. The NEB method locates the transition state between relaxed initial (the dislocation dipole) and final (a perfect fcc lattice) states; here we use a linear interpolation in all atomic coordinates as an initial guess. The transition path is represented computationally by a sequence of 25 configurations between the initial and final configurations. The path is subsequently relaxed to a so-called minimum energy path, where the force perpendicular to the path vanishes. The saddle point configuration (transition state) is then the configuration with maximal energy along the minimum energy path. The NEB calculation leads to a value of $E=315 \mathrm{meV}$ for the transition state energy, consistent with the value of the activation energy obtained by Arrhenius fitting to the MD results.

Having seen that the activation energy calculated from the MD simulations agrees well with the expectation from TST we continue to discuss the obtained rate prefactor in the same framework. In harmonic TST the entropic contribution to the rate [Eq. (2)] is calculated by performing a harmonic approximation to the potential energy surface around the initial state and the transition state [6]. The prefactor then becomes independent of temperature, and it is given by the frequencies $\omega_{i}$ of the eigenmodes at the transition state (TS) and in the initial state (IS),

$$
\nu=\frac{1}{2 \pi} \frac{\prod_{i} \omega_{i}^{\mathrm{IS}}}{\prod_{i}^{\prime} \omega_{i}^{\mathrm{TS}}}
$$

where the prime indicates that the mode with imaginary frequency corresponding to the reaction coordinate has been left out of the product over the TS modes. The harmonic approximation can be expected to work well at sufficiently low temperatures, where all modes can be considered harmonic (excluding possible quantum effects at very low temperatures). In this low temperature regime the computational unit cell must actually contain 30 identical transition states on each dislocation, which can be transformed into each other by translation with an integer times the Burgers vector. Each of these transition states would give the same contribution to the rate so-pretending for a moment that the simulations are in this low temperature regime - the proper prefactor from a single transition state would be $\nu / 60=4 \times 10^{13} \mathrm{sec}^{-1}$. This value is within 1 order of magnitude of other atomistic prefactors obtained, for example, for single-atomic diffusion processes [3].

The harmonic approximation should not in fact be considered applicable for all of the relevant modes in the investigated temperature range. If we, for example, consider the translation of the transition state along the direction of the dislocation line, the energy barrier is probably of the same order of magnitude or smaller than the barrier for jog mobility on single screw dislocations, which was recently calculated to be only $15-20 \mathrm{meV}$ [16]. This value is similar to the temperature, so the harmonic approximation is bound to fail. In the opposite limit, where the temperature is much higher than the energy barriers separating the different transition states, a simple calculation of the entropy shows [17] that, in Eq. (3), the frequency corresponding to this mode should be replaced by $\sqrt{2 \pi k_{B} T / m b^{2}}$. A power-law dependence on the temperature thus appears in the prefactor. Unfortunately, it is not possible to determine the exact temperature dependence of the prefactor from the MD simulations because of the limited temperature range available.

It is interesting to compare the calculated prefactor with simple estimates based on dislocation theory. If we first consider a single screw dislocation split up into partials, a natural "attempt frequency" for cross slip would be the frequency $\omega_{0}$ corresponding to an oscillation in the separation between straight partials. From linear elasticity theory this frequency is seen to be [10] $\omega_{0}=$ $\sqrt{\mu / 16 \pi \rho^{*}}(b / d)$, where $\mu$ is the shear modulus along the closed packed $\{111\}$ planes, $d$ is the separation between the partials, and $\rho^{*}$ is the dislocation mass (per length of dislocation). Taking $\mu=44 \mathrm{GPa}$ (from the EMT potential) and $d=3 b$ (from the simulations), and obtaining a rough estimate of the mass as $\rho^{*}=\rho_{0} b^{2} / 4 \pi=$ $4.6 \times 10^{-17} \mathrm{~kg} / \mathrm{m}$ (see Ref. [10]), we find a frequency of $\omega_{0}=1.5 \times 10^{12} \mathrm{sec}^{-1}$. However, this frequency cannot be directly compared with the prefactor per length obtained from the MD simulations as we are still missing the characteristic length.

To see how the characteristic length appears in a dislocation description of the rate and in order to provide a rough estimate of the entropic contributions from fluctuations of the dislocation lines we shall consider a simplified model, which has the virtue of being exactly solvable. The model contains a string described by a function $y(x, t)$, where $x$ is a space coordinate and $t$ is the time. The Lagrangian $L$ for the string is given by

$$
L=\int\left[\frac{1}{2} \rho^{*}\left(\frac{\partial y}{\partial t}\right)^{2}-\frac{1}{2} \alpha\left(\frac{\partial y}{\partial x}\right)^{2}-V(y(x))\right] d x,
$$

with a quartic double-well potential $V(y)=\frac{\rho^{*} \omega_{0}^{2}}{8 d^{2}}(y-$ $d)^{2}(y+d)^{2}$. The model can be viewed as describing the cross slip of a screw dislocation using a line tension description of the dislocation lines. The variable $y$ corresponds to the separation between the two partials. In the initial state the partials are separated by a distance $d$ in the primary glide plane (corresponding to the solution $y=d$ ), and after the cross slip they have the same separation in the cross slip plane (corresponding to $y=-d$ ). The quartic potential is chosen so that the frequency for the straight string vibrating around the $y=d$ solution is $\omega_{0}$. The transition state of the cross slip process in this model consists of a widely separated kink-antikink pair with the reaction coordinate being the distance between the kink and the antikink. (A "kink" in the model corresponds to a "constriction" in the real cross slip process.) The beauty of the model is that it is possible to calculate the harmonic prefactor [Eq. (3)] exactly [18,19]. We shall not repeat the rather lengthy calculations here, but just state the simple 
result. For a string configuration with a single kink (i.e., where $y(x) \rightarrow \pm d$ for $x \rightarrow \pm \infty$ ) the fluctuation factor is surprisingly simple: $F \equiv \prod_{i} \omega_{i}^{\mathrm{IS}} / \prod_{i}^{\prime} \omega_{i}^{\text {kink }}=\sqrt{12} \omega_{0}$, where the prime indicates that the eigenmode corresponding to translation of the kink has been omitted.

Returning to the kink-antikink pair as the transition state, we see that we have to treat the two modes related to kink and antikink translation in a special way. The translation of the kink and antikink relative to each other is the reaction coordinate, which should be omitted from the product over modes in Eq. (3). The other mode corresponds to the common translation of the kink and antikink together, and for symmetry reasons this mode has zero eigenfrequency. As a consequence the prefactor becomes proportional to the length of the string. In fact we can use the above-mentioned substitution rule for a free mode $\omega \rightarrow \sqrt{2 \pi k_{B} T / M \ell^{2}}$, where the length $\ell$ of the dislocation now enters and $M$ is the mass corresponding to kink translation. The final result for the prefactor becomes

$$
\nu_{\text {string }}=\ell \frac{\omega_{0}}{2 \pi} 12\left(\frac{M \omega_{0}^{2}}{2 \pi k_{B} T}\right)^{1 / 2} \text {. }
$$

Inserting $M=M_{\mathrm{Cu}}$ as the mass for the constriction of the partials [16] and $T=300 \mathrm{~K}$, this corresponds to a prefactor of $\nu_{\text {string }}=1.3 \times 10^{14} \mathrm{sec}^{-1}$, which is only an order of magnitude from the result of our simulations.

In conclusion we have determined the temperature dependent rate for screw dislocation cross slip by direct molecular dynamics simulations of the annihilation of a screw dislocation dipole. The rate is found to obey an Arrhenius behavior, and the activation energy is shown to agree with the transition state energy. The prefactor is shown to have a typical atomic-scale value, as also expected from a simple line tension dislocation model.

The Center for Atomic-scale Materials Physics (CAMP) is sponsored by the Danish National Research Foundation. The present work was in part financed by The Danish Research Councils through Grant No. 9501775 and was done as a collaboration between CAMP and the Engineering Science Centre for Structural Characterization and Modelling of Materials at the Materials Research Department, Ris $\varnothing$.

[1] V. Bulatov, F. F. Abraham, L. Kubin, B. Devincre, and S. Yip, Nature (London) 391, 669 (1998).
[2] J. Schiøtz, F. D. Di Tolla, and K. W. Jacobsen, Nature (London) 391, 561 (1998); H. Van Swygenhoven, M. Spaczer, A. Caro, and D. Farkas, Phys. Rev. B 60, 22 (1999).

[3] H. Brune, Surf. Sci. Rep. 31, 121 (1998); T. Itoh, G. R. Bell, A. R. Avery, T. S. Jones, B. A. Joyce, and D. D. Vvedensky, Phys. Rev. Lett. 81, 633 (1998); C. L. Liu, J. M. Cohen, J. B. Adams, and A. F. Voter, Surf. Sci. 253, 334 (1991).

[4] B. Devincre and L. P. Kubin, Mater. Sci. Eng. A 234-236, 8 (1997).

[5] A.F. Voter, Phys. Rev. Lett. 78, 3908 (1997); M. R. Sørensen and A.F. Voter, J. Chem. Phys. 112, 9599 (2000).

[6] P. Hänggi, P. Talkner, and M. Borkovec, Rev. Mod. Phys. 62, 251 (1990); A. F. Voter and J. Doll, J. Chem. Phys. 82, 80 (1985).

[7] G. Boisvert, N. Mousseau, and L. J. Lewis, Phys. Rev. B 58, 12667 (1998); U. Kürpick and T. S. Rahman, Surf. Sci. 427, 15 (1999).

[8] T. Rasmussen, K. W. Jacobsen, T. Leffers, O. B. Pedersen, S. G. Srinivasan, and H. Jónsson, Phys. Rev. Lett. 79, 3676 (1997).

[9] K. W. Jacobsen, J. K. Nørskov, and M. J. Puska, Phys. Rev. B 35, 7423 (1987); K. W. Jacobsen, P. Stoltze, and J. K. Nørskov, Surf. Sci. 366, 394 (1996), and references therein.

[10] J.P. Hirth and J. Lothe, Theory of Dislocations (Krieger, Malabar, 1992), 2nd ed.

[11] N. M. Rosengaard and H. L. Skriver, Phys. Rev. B 47, 12865 (1993).

[12] T. Rasmussen, T. Vegge, K. W. Jacobsen, T. Leffers, and O. B. Pedersen, Philos. Mag. A 80, 1273 (2000).

[13] P. Stoltze, Simulation Methods in Atomic-Scale Materials Physics (Polyteknisk forlag, Lungby, 1997).

[14] H. Jónsson and H. C. Andersen, Phys. Rev. Lett. 60, 2295 (1988); A. S. Clarke and H. Jónsson, Phys. Rev. E 47, 3975 (1993).

[15] G. Mills, H. Jónsson, and G. Schenter, Surf. Sci. 324, 305 (1995); H. Jónsson, G. Mills, and K. W. Jacobsen, Classical and Quantum Dynamics in Condensed Phase Simulations, edited by B. J. Berne, G. Ciccotti, and D. F. Coker (World Scientific, Singapore, 1998).

[16] T. Vegge, O. B. Pedersen, T. Leffers, and K. W. Jacobsen, Mater. Sci. Eng. A (to be published).

[17] A configuration space integral over a harmonic degree of freedom $q$ gives $\int \exp \left(-m \omega^{2} q^{2} / 2 k_{B} T\right) d q=$ $\sqrt{2 \pi k_{B} T / m \omega^{2}}$, whereas an integral over a free degree of freedom over the length $b$ gives $\int d q=b$. Thus the substitution rule $\omega \rightarrow \sqrt{2 \pi k_{B} T / m b^{2}}$.

[18] S. Coleman, Phys. Rev. D 15, 2929 (1977).

[19] H. Kleinert, Path Integrals in Quantum Mechanics and Polymer Physics (World Scientific, Singapore, 1995), 2nd ed. 